Article

\title{
High-Precision Hysteresis Sensing of the Quartz Crystal Inductance-to-Frequency Converter
}

\author{
Vojko Matko ${ }^{1, *}$ and Miro Milanović ${ }^{2}$ \\ 1 Institute for Automation, Faculty of Electrical Engineering and Computer Science, University of Maribor, \\ Smetanova 17, Maribor 2000, Slovenia \\ 2 Institute for Robotics, Faculty of Electrical Engineering and Computer Science, University of Maribor, \\ Smetanova 17, Maribor 2000, Slovenia; miro.milanovic@um.si \\ * Correspondence: vojko.matko@um.si; Tel.: +386-2-220-7111
}

Academic Editor: Stephane Evoy

Received: 29 April 2016; Accepted: 18 June 2016; Published: 28 June 2016

\begin{abstract}
A new method for the automated measurement of the hysteresis of the temperature-compensated inductance-to-frequency converter with a single quartz crystal is proposed. The new idea behind this method is a converter with two programmable analog switches enabling the automated measurement of the converter hysteresis, as well as the temperature compensation of the quartz crystal and any other circuit element. Also used is the programmable timing control device that allows the selection of different oscillating frequencies. In the proposed programmable method two different inductances connected in series to the quartz crystal are switched in a short time sequence, compensating the crystal's natural temperature characteristics (in the temperature range between 0 and $50^{\circ} \mathrm{C}$ ). The procedure allows for the measurement of the converter hysteresis at various values of capacitance connected in parallel with the quartz crystal for the converter sensitivity setting at selected inductance. It, furthermore, enables the measurement of hysteresis at various values of inductance at selected parallel capacitance (sensitivity) connected to the quartz crystal. The article shows that the proposed hysteresis measurement of the converter, which converts the inductance in the range between 95 and $100 \mu \mathrm{H}$ to a frequency in the range between 1 and $200 \mathrm{kHz}$, has only $7 \times 10^{-13}$ frequency instability (during the temperature change between 0 and $50^{\circ} \mathrm{C}$ ) with a maximum $1 \times 10^{-11}$ hysteresis frequency difference.
\end{abstract}

Keywords: inductance-to-frequency converter; hysteresis; sensor switching method; inductance; temperature compensation

\section{Introduction}

Nowadays, inductance-to-frequency conversion has become increasingly used in a large variety of applications that are designed for the measurement of a number of physical measurands, such as mechanical displacement (position), pressure, nanopositioning, strain sensing, eccentric motion, biosensors, in medical and electromagnetic material properties measurements, and quartz crystal microbalance [1-3]. High-resolution inductance-to-frequency conversion, in particular, is a well-established technique in microscale converters for material properties sensing. It represents a universal transduction mechanism for the measurements in which the inductance changes need to be measured with great precision. The hysteresis of the converter in such measurements plays a very important role.

Recent research studies have focused mainly on the sensor methods that would make precise measurements with the help of an inductance change in the range well below some $\mu \mathrm{H}$ possible. In such measurements both inductive resolution and inductance-to-frequency converter hysteresis play a vital role. The problem of temperature drift was addressed by one of the studies with an 
analog-digital mixed-measurement method based on the two-dimensional look-up table. Designed for inductive proximity sensors (IPS) widely used in position detection, the method reduced the measurement error caused by temperature drift, but unlike this study it works in the $\mathrm{mH}$ inductive range and is not linear [4]. Change in the sensor's resonant frequency can also be used to detect the pressure wirelessly. In the implantable passive LC pressure sensor proposed by an interesting study, the inductance and capacitance elements of the sensor were designed independently and separated by a thermally-insulating material, which was conducive to reducing the influence of the temperature on the inductance element of the sensor. The linearity and repeatability errors achieved were $95.3 \%$, and $5.5 \%$, respectively, as a function of pressure at $800^{\circ} \mathrm{C}$ [5]. Apart from temperature, passive LC sensors can be used to monitor pressure and harmful gases in harsh environments. The paper discusses the advantages and disadvantages of various sensor types and establishes that, for the measurement of multifunction sensors (because of the mutual inductance among multiple parameters), precise readout of multiple parameters requires a complex decoupling method. In addition, current passive LC sensors exhibit some degree of temperature drift [6]. With silicon's better thermal material properties, silicon micromachined pressure sensors are widely used. The thermal mismatching between the sensing element and packaging may generate stresses on the transducer of a sensing element and create thermal hysteresis and voltage shift during temperature cycling (a very low voltage is involved). In the paper, finite element analyses and experimental tests were conducted to reduce the thermal stress and thermal hysteresis for differential pressure sensors [7].

Other research used a displacement-to-frequency transducer based on the variation of a coil inductance when a magnetic core is partially or completely inserted inside. Studies of the thermal stability of the transducer have been performed with the maximum frequency variation of $24 \mathrm{~Hz}$-equivalent to $21 \mu \mathrm{m}$ (below the sensor accuracy $33.5 \mathrm{~Hz}$-equivalent to $28 \mu \mathrm{m}$ ). However, no temperature analysis of the influence of the flexible core on the frequency variation was made [8]. The sensing principle of an ink-jet printed eddy current position sensor has been thoroughly studied and validated by a paper that used both simulations and measurements. The results showed that the design of the sensor is very dependent on the limitations of the fabrication, especially the printing of conductors. Additionally, the temperature influence of the oscillator has not been taken into account [9]. Another paper presents a temperature and strain monitoring system for a magnetic actuator based on the magnetostrictive material Terfenol-D (Tb0.3 Dy0.7Fe1.92) with four fiber Bragg sensors. This system allows making the appropriate hysteresis compensation on the strain measurement due to temperature drift. The paper, however, does not analyze the temperature hysteresis influence of the electronic amplifier circuit and fiber Bragg sensor [10]. Small inductance changes measured at the frequency of $4.999 \mathrm{MHz}$ can be detected with high sensitivity in the temperature range between $10^{\circ} \mathrm{C}$ to $40{ }^{\circ} \mathrm{C}$. The main advantage of this method lies in a considerable reduction of the temperature influence of the AT-cut crystal frequency change in the temperature range between $10{ }^{\circ} \mathrm{C}$ and $40{ }^{\circ} \mathrm{C}$ through a switching method. The method, however, has not researched the hysteresis influence brought about by the switching method [11]. The above methods looked for ways either of how to detect temperature influence or how to compensate it, but they have not made any significant analysis with regard to the sensors' (electronic circuits) hysteresis influence on the measurement error.

The novelty in this work is the automated measurement of the quartz crystal inductance-to-frequency converter characteristics and hysteresis through the switching between the measuring inductance and the reference one using an analog multiplexer (MUX) switch. To set the capacitance in the first place, an analog single-pole single-throw (SPST) switch for the switching of additional parallel capacitances to the quartz crystal is used. In addition, a programmable switching control device for the converter hysteresis measurement was added. The use of all of these elements improves the quality of converter characteristics and hysteresis measurement, the frequency sensitivity of the converter, compensates the quartz crystal non-linear self-temperature frequency characteristic, as well as any parasitic capacitances of the oscillator circuit, and enables a very stable converter functioning in an extended temperature range. A switching mode oscillator, furthermore, strongly 
reduces the influence of the supply voltage on the output signal oscillating frequency, and foresees the functioning of the sensitive inductance element.

Additional comparisons to some other methods [12-14] for the conversion of inductance to frequency and hysteresis detection reveal that the newly-proposed method also proves to have high dynamic stability during the temperature changes in the extended operating range when the temperature varies between 0 and $50{ }^{\circ} \mathrm{C}$. With regard to the temperature, the method makes possible a stable functioning of small inductance change measurement and conversion to a frequency signal (without any additional lock-in amplifier, host system, or temperature sensor).

\section{Switching Mode Inductance-to-Frequency Converter}

\subsection{Switching Converter Principle}

A quartz crystal inductance-to-frequency converter uses the principle of switching between two inductances connected in series, changing in this way the quartz crystal series resonance frequency. The purpose of this switching is the temperature compensation of the serial resonance frequency, which is temperature-dependent due to the quartz temperature dependency. It additionally compensates any other influence, such as parasitic capacitances and inductances, aging of the elements, etc., with the help of an additional reference inductance [11].

The operation of a quartz crystal (frequently explained using the familiar "Equivalent Circuit") is illustrated in Figure 1a,b representing an electrical depiction of the quartz crystal unit [15-17]. By connecting the inductance $L_{\mathrm{L}}$ with its own resistance $R_{\mathrm{L}}$, the quartz crystal series resonance frequency can be changed from 0 to $200 \mathrm{kHz}$.
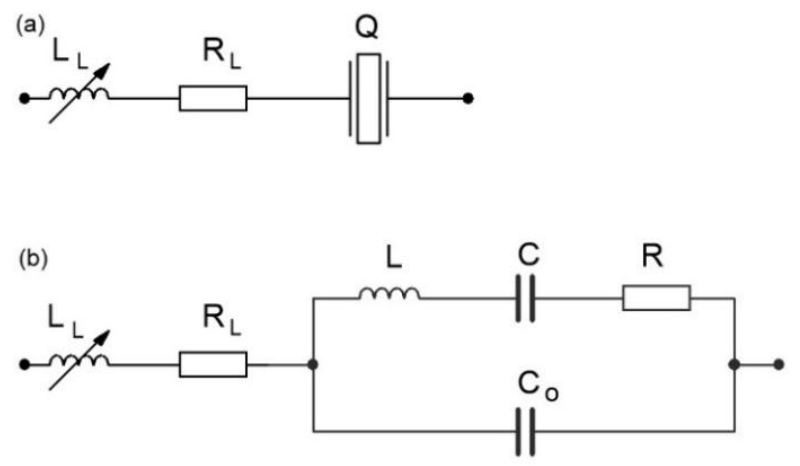

Figure 1. (a) The real inductance $L_{\mathrm{L}}+R_{\mathrm{L}}$ in series with the quartz crystal and (b) the real inductance $L_{\mathrm{L}}+R_{\mathrm{L}}$ with the quartz crystal equivalent circuit.

The quartz series resonance frequency without the inductance $L_{\mathrm{L}}$ (which has its own resistance $R_{\mathrm{L}}$ ) connected in series is determined by Equation (1):

$$
f_{0}=\frac{1}{2 \pi \cdot \sqrt{L \cdot C}}
$$

For the connection of the quartz crystal with inductance $L_{\mathrm{L}}$ in series, an equation with equivalent impedance can be written. If we define the frequency ratio $\Omega=\omega / \omega_{0}$, which depends on $\omega_{\mathrm{o}}=1 / \sqrt{L \cdot C}$, and take into account $\omega_{\mathrm{o}} L=1 / \omega_{\mathrm{o}} C$, then the impedance change in the vicinity of the resonance frequency is described by Equation (2) in the range of change $\Omega=0.998-1.038$ [15-17]:

$$
\bar{Z}(\Omega)=R \frac{1+j \frac{\omega_{0} L}{R}\left(\Omega-\frac{1}{\Omega}\right)}{1+\frac{C_{0}}{C}\left(1-\Omega^{2}\right)+j \frac{C_{0}}{C} \cdot \frac{R}{\omega_{0} L} \cdot \Omega}+j \frac{\Omega \cdot L_{L}}{\sqrt{L \cdot C}}+R_{L}
$$


Figure 2 depicts the oscillator circuit switching between the reference inductance $L_{\text {ref }}$ in series with the quartz crystal and the measuring inductance $L_{\mathrm{L}}$ in series with the quartz crystal. The switching between these two inductances produces two new resonance frequencies $f_{01}$ and $f_{02}$ at the output. Switching signals $Q$ and $\bar{Q}$ are digital signals 1 or 0 .

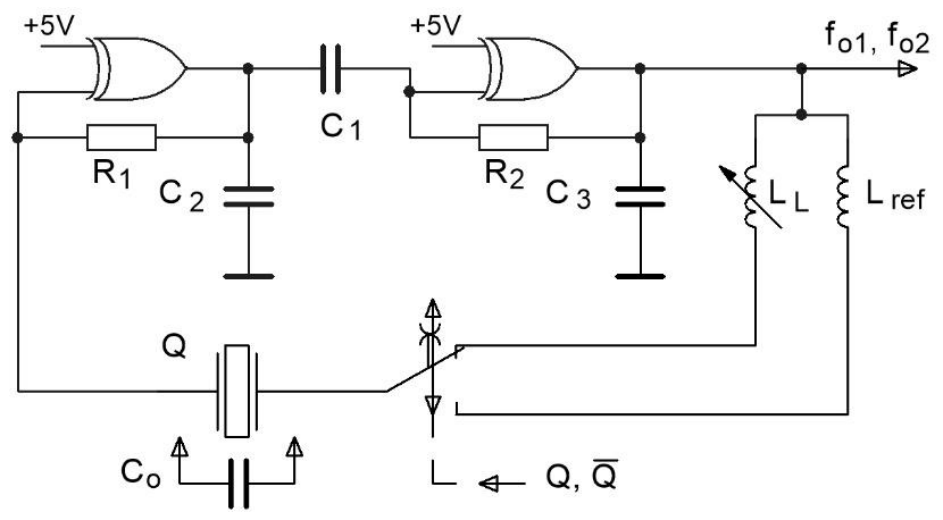

Figure 2. Switching of the oscillator inductances in the inductance-to-frequency converter.

The pulling range between the switching of inductances $L_{\mathrm{L}}$ and $L_{\text {ref }}$ with the signals $Q$ and $\bar{Q}$ (Figure 2) can be written with Equation (3) [15-17]:

$$
D_{L_{L}, L_{r e f}}=\frac{f_{01 L_{L}}-f_{02 L r e f}}{f_{02 L r e f}}
$$

The change of resonance frequency $f_{01}$ (Figure 2) produced by the influence of inductance $L_{\mathrm{L}}$ is expressed with Equation (4) [15-17]:

$$
f_{01}=\frac{1+\frac{C}{2\left(C_{0}-\frac{1}{\omega_{0}^{2} \cdot L_{L}}\right)}}{2 \pi \cdot \sqrt{L \cdot C}}
$$

The change of resonance frequency $f_{02}$ (Figure 2) produced by the influence of inductance $L_{\text {ref }}$ is described in Equation (5) [15-17]:

$$
f_{02}=\frac{1+\frac{C}{2\left(C_{0}-\frac{1}{\omega_{0}^{2} \cdot L_{r e f}}\right)}}{2 \pi \cdot \sqrt{L \cdot C}}
$$

\subsection{Converter Hysteresis Measurement Principle}

The new idea of the converter hysteresis measurement method described in this article is the use of a specific switching mode oscillator with additionally-connected inductances in series with the quartz crystal alternating between $L_{1}-L_{7}$ and $L_{\text {ref }}$ (Figure 3 ). Additional shunt capacitances $C_{10}-C_{12}$ are connected together with the SPST analog switch in parallel to the quartz crystal for the experimental stepwise increase (sensitivity settings) of the capacitance $C_{0}$. Conversion inductances $L_{1}-L_{7}$ and reference inductance $L_{\text {ref }}$ are connected to the quartz crystal alternately (switching mode) in series. They enable a significant reduction of the temperature influence (both are on the same temperature) on the oscillator frequency change because of the switching mode oscillator. Capacitance $C_{\text {set }}$ is used here for accurate setting of the initial frequency of the oscillator. To switch the frequencies from $f_{01}$ to $f_{07}$ the NI myDAQ (National Instruments, Austin, TX, USA) programmable device and the LabVIEW (LW) software (National Instruments, Austin, TX, USA) are used. The switching is performed through the switching (switching table of the eight-channel analog MUX switch) of digital 
signals (wires $\mathrm{S}_{1}-\mathrm{S}_{3}$ ). With the help of the reference frequency $f_{\mathrm{r}}$ (from an oven-controlled crystal oscillator (OCXO18T5S)), pulse width module (PWM), and low-pass filter (LP) frequencies $f_{01-07}$

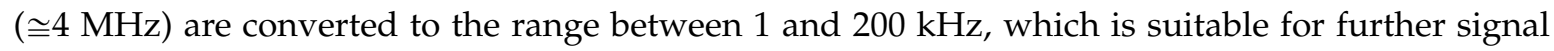
processing. The signal corresponding to the frequency difference between the oscillator frequencies $f_{01-07}$ and reference frequency $f_{\mathrm{r}}$ enters the LP filter (which is a pulse width modulated signal) [18-20]. At the passive LP filter (with the response time of $3 \mu \mathrm{s}$ ) output, the triangular signal (with the initial setting frequency of $5 \mathrm{kHz}$ depending on $C_{\text {set }}, L_{\text {ref, }}$ and $f_{\mathrm{r}}=4 \mathrm{MHz}$ ) is produced (frequency difference between $f_{0 \text { Lref }}$ and $f_{\mathrm{r}}$ ). This signal is then converted to a rectangular signal by a signal transforming circuit representing the output signal $f_{\text {out }}$ of the inductance-to-frequency converter. The output frequency $f_{\text {out }}$ thus represents the temperature and any other influence-compensated signal which is synchronously measured with regard to the switching frequency. The latter can change in the range $f_{\text {Switch }}=1-20 \mathrm{~Hz}$ [10]. Capacitances $C_{2}$ and $C_{3}$ serve to suppress the spurious responses to avoid crystal oscillation at higher frequencies, and $C_{4}$ reduces the high frequency noise of the oscillator in the channel A of the HM 8122 programmable counter [21]. Channel A of the programmable counter measures the frequency $f_{01-07}$ in relation to the digital signals (wires $S_{1}-S_{3}$ ), while channel B measures the frequency difference $f_{\text {out }}=f_{01-07}-f_{\mathrm{r}}$ or $f_{0 \text { Lref }}-f_{\mathrm{r}}$ depending on the switching digital state (wires $\mathrm{S}_{1}-\mathrm{S}_{3}$ ).

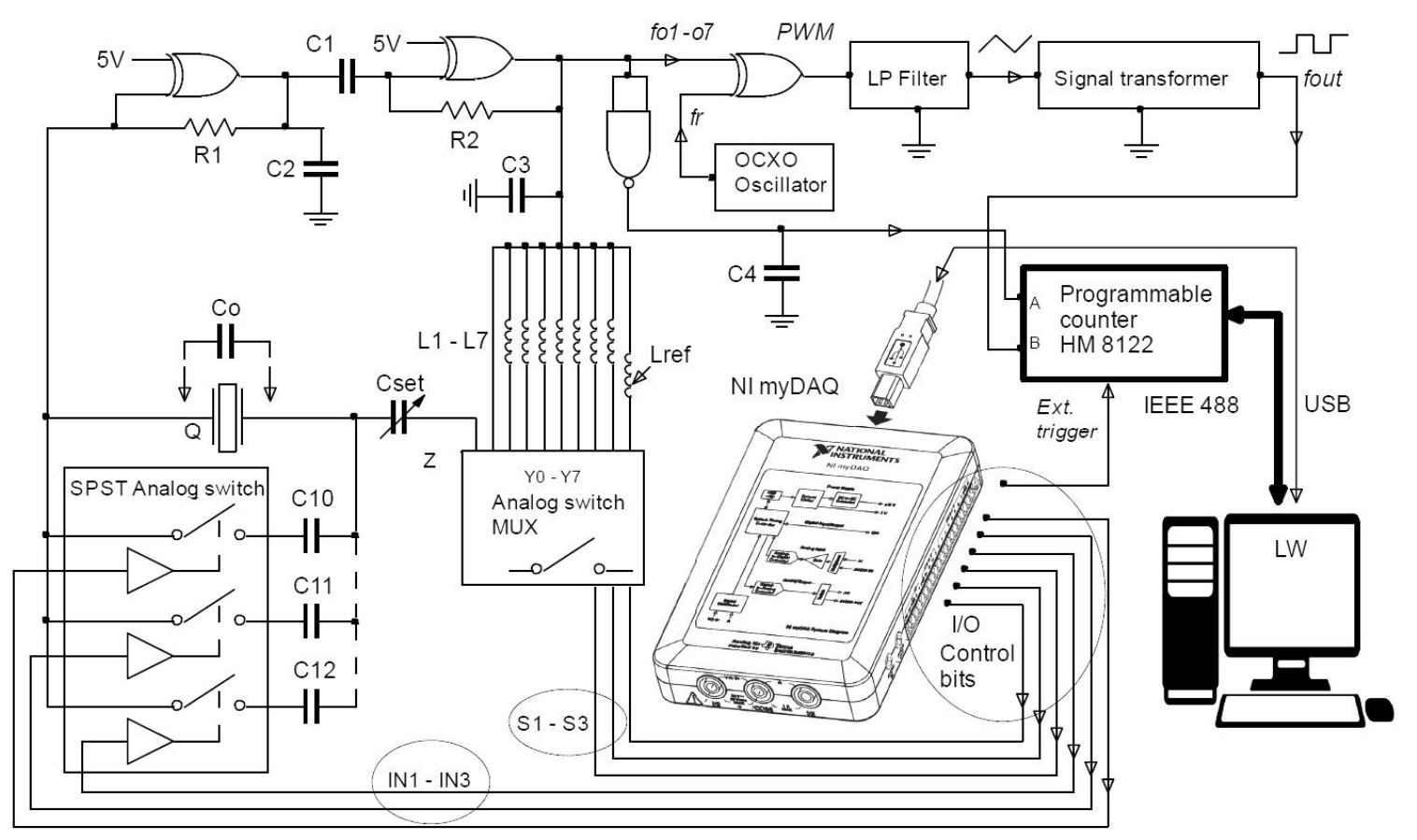

Figure 3. Schematic representation of the inductance-to-frequency converter hysteresis measurement.

For every switching between one of the inductances $L_{1-7}$ and $L_{\text {ref }}$ (by changing digital signals sent by wires $S_{1}-S_{3}$ ), a positive impulse from NI myDAQ triggers the frequency measurement on the counter (external trigger). In this way, the frequency counter measures frequency synchronously with the LW programmable digital signal changes (wires $S_{1}-S_{3}$ ). Moreover, the LW software driver also makes it possible to statistically evaluate the number of performed consecutive measurements.

The quartz stray capacitance $C_{0}$ comprises pin-to-pin parallel capacitance at the crystal pins, plus any parasitic capacitances. The typical value of the quartz stray capacitance is between $1.5 \mathrm{pF}$ and $5 \mathrm{pF}$. The connection of an additional stray capacitance connected in parallel (by SPST analog switch controlled by digital signals sent by wires $\mathrm{IN}_{1}-\mathrm{IN}_{3}$ ) to the quartz crystal for capacitive load settings, and of inductance $L_{1-7}$ in series with the quartz crystal expands the possibility of the measurement of 
the inductance-to-frequency converter hysteresis where the stable oscillation and high sensitivity are one of this method's major advantages [22-24].

\subsection{Temperature Compensation}

Temperature compensation of the inductance-to-frequency converter is achieved via the oscillator's switching method, which reduces the influence of short-term temperature instability and long-term instability (aging), representing the oscillator frequency variation as a function of time. The short-term stability of a quartz crystal depends on the actual oscillator design and is totally controlled by the quartz crystal at a low drive level $(<10 \mu \mathrm{W})$ [25]. Long-term stability changes with years and is naturally greater during the first part of the crystal unit life. The aging rates of the best cold weld crystals are less than $\pm 1 \mathrm{ppm} /$ year $\left(0-50^{\circ} \mathrm{C}\right)$ [22].

The principle of temperature compensation is explained below through the example of two switchings between the inductances $L_{\mathrm{L}}$ and $L_{\text {ref }}$ (as illustrated on Figure 2), where inductance $L_{\mathrm{L}}$ represents the inductance $L_{1}$ in Figure 3 [11]. When inductances $L_{1}$ and $L_{\text {ref }}$ are the same, $f_{01}$ and $f_{02}$ remain almost the same depending on the quartz crystal resonant frequency $f_{0}$, quartz crystal temperature characteristics $\Delta f_{0}(T)$, its aging $\Delta f_{0}(t)$, and the $L_{1}$ and $L_{\text {ref }}$ inequality, as well as the $\Delta f_{0}$ $\left(\Delta C_{0 \mathrm{p}}\right)$ change. However, when inductances $L_{1}$ and $L_{\text {ref }}$ are different, the frequencies $f_{01}$ and $f_{02}$ are different and depend on the quartz crystal series resonant frequency $f_{0}$, quartz crystal temperature characteristics $\Delta f_{0}(T)$, its aging $\Delta f_{0}(t)$, inductances $\Delta f_{0}\left(L_{2}\right)$ and $\Delta f_{0}\left(L_{\text {ref }}\right)$, as well as the $\Delta f_{0}\left(\Delta C_{0 \mathrm{p}}\right)$ change. In the case of the difference of the two frequencies $f_{01}$ and $f_{02}, \Delta f_{0}(T), \Delta f_{0}(t)$, and $\Delta f_{0}\left(\Delta C_{0 \mathrm{p}}\right)$ are strongly reduced because only one temperature quartz characteristic is involved [11].

$f_{0}$-quartz crystal series resonant frequency

$f_{\mathrm{r}}$-reference frequency

$\Delta f_{\mathrm{c}}$-counter error

$L$ and $C$ - mechanical behavior of the crystal element

$L_{\mathrm{n}}$ - conversion inductance $(n=1-7)$

$L_{\text {ref }}$-reference inductance

$\mathrm{C}_{0}$ - quartz crystal parallel capacitance

$\mathrm{C}_{0 \mathrm{p}}$-all actual parallel parasitic capacitances connected to the quartz crystal

T-temperature

$t$ - time

The output frequency $f_{\text {out }}$ (Figure 3 ) depends on the selection of digital signals sent by wires $\mathrm{S}_{1}-S_{3}$ (frequency $f_{01-07}$ ) and reference frequency $f_{\mathrm{r}}$ and can be expanded to (in case $Z_{1}=R_{1}+\mathrm{j} \omega L_{1}$ to $Z_{7}=R_{7}+j \omega L_{7}$ and $Z_{\text {ref }}=R_{\text {ref }}+j \omega L_{\text {ref }}$, whereby when dealing with small inductance values, resistances $R_{1-7}$ and $R_{\text {ref }}$ can be ignored):

$$
\begin{gathered}
f_{\text {out } 1}=f_{01}-f_{r}=f_{0}+\Delta f_{0}\left(T_{1}\right)+\Delta f_{0}\left(t_{1}\right)+\Delta f_{0}\left(C_{0 p}\right)+\Delta f_{0}\left(L_{n}\right)-\left(f_{r}\left(T_{1}\right)+\Delta f_{r}\left(T_{1}\right)\right)+\Delta f_{c 1}\left(t_{1}\right) \\
f_{\text {out } 2}=f_{02}-f_{r}=f_{0}+\Delta f_{0}\left(T_{2}\right)+\Delta f_{0}\left(t_{2}\right)+\Delta f_{0}\left(C_{0 p}\right)+\Delta f_{0}\left(L_{r e f}\right)-\left(f_{r}\left(T_{2}\right)+\Delta f_{r}\left(T_{2}\right)\right)+\Delta f_{c 2}\left(t_{2}\right)
\end{gathered}
$$

where $\Delta f_{\mathrm{r}}(\mathrm{T})$ in Equations (6) and (7) represents the temperature instability of the reference oscillator signal and $\Delta f_{\mathrm{c}}(t)$ a counter error [16,17]. The joining of $f_{0}$ and $\Delta f_{0}\left(L_{\mathrm{n}}\right)$ gives Equation (8). Which represents $f_{01}$ :

$$
\begin{gathered}
f_{01}=\frac{1+\frac{C}{2\left(C_{0 p}-\frac{1}{\omega_{0}^{2} \cdot L_{n}}\right)}}{2 \pi \cdot \sqrt{L \cdot C}}+\Delta f_{0}\left(T_{1}\right)+\Delta f_{0}\left(t_{1}\right) \\
\omega_{0}=2 \pi f_{0}
\end{gathered}
$$


The joining of $f_{0}$ and $\Delta f_{0}\left(L_{\text {ref }}\right)$ gives Equation (10) which represents $f_{02}$ :

$$
f_{02}=\frac{1+\frac{C}{2\left(C_{0 p}-\frac{1}{\omega_{0}^{2} \cdot L_{r e f}}\right)}}{2 \pi \cdot \sqrt{L \cdot C}}+\Delta f_{0}\left(T_{2}\right)+\Delta f_{0}\left(t_{2}\right)
$$

At every switch between two inductances, the frequency $f_{\text {out }}$ is measured synchronously by the HM 8122 programmable counter (Figure 3) and its value is transferred to the LW software calculating the difference between the two frequencies. This gives the frequency difference in Equations (11) and (12), representing the temperature-compensated value of the output frequency $f_{\text {out }}$, depending almost uniquely on the difference between the $\Delta L_{n}$ and $\Delta L_{\text {ref }}$ change. This means that $\Delta f_{\text {out }}\left(L_{n}\right)$ is virtually independent of the quartz crystal temperature characteristics $\Delta f_{0}(T)$. The quartz aging $\Delta f_{0}$ $(t)$ is practically compensated and can be ignored as the measurements are short and consecutive (a few milliseconds). Due to the switching of the frequencies $f_{01}$ and $f_{02}$, the output frequency $f_{\text {out }}$ also highly reduces the auxiliary reference frequency $f_{\mathrm{r}}$ temperature instability $\Delta f_{\mathrm{r}}(T)$ :

$$
\Delta f_{\text {out }}\left(L_{n}\right)=\left[f_{01}-\left(f_{r}\left(T_{1}\right)+\Delta f_{r}\left(T_{1}\right)+\Delta f_{c 1}\left(t_{1}\right)\right)\right]-\left[f_{02}-\left(f_{r}\left(T_{2}\right)+\Delta f_{r}\left(T_{2}\right)+\Delta f_{c 2}\left(t_{2}\right)\right)\right]
$$

The reference frequency $f_{\mathrm{r}}$ is OCXO18T5S oven-controlled crystal oscillator $(4 \mathrm{MHz})$ with the frequency stability of $\pm 0.01 \mathrm{ppm}$ in the temperature range $0^{\circ} \pm 60^{\circ} \mathrm{C}$ following the warm-up time of $1 \mathrm{~min}$ [25-27]. The $f_{\mathrm{r}}(T)$ is compensated in Equation (11). Equation (12) is formed taking into account that the counter error $\Delta f_{\mathrm{c}}(t)$ is different at every switching for the time $t_{1}$ and $t_{2}$ :

$$
\Delta f_{\text {out }}\left(L_{n}\right)=\left[\frac{\frac{c}{2\left(c_{0 p}-\frac{1}{\omega_{0} \cdot L_{L}}\right)}}{2 \pi \cdot \sqrt{L \cdot C}}\right]-\Delta f_{0}\left(T_{1}\right)-\Delta f_{r}\left(T_{1}\right)+\Delta f_{c 1}\left(t_{1}\right)-\left[\frac{\frac{c}{2\left(c_{0 p}-\frac{1}{\omega_{0}^{2} \cdot L_{\text {ref }}}\right)}}{2 \pi \cdot \sqrt{L \cdot C}}\right]+\Delta f_{0}\left(T_{2}\right)+\Delta f_{r}\left(T_{2}\right)-\Delta f_{c 2}\left(t_{2}\right)
$$

The proposed method (Figure 3) allows the AT-cut crystal temperature characteristics compensation (under $0.00001 \mathrm{~Hz}$ ) in the range between 0 and $50{ }^{\circ} \mathrm{C}$ for the crystal cut angle $0^{\prime}$ through the switching circuit and significantly reduces its influence to a minimum.

\subsection{The Shortcoming of the Temperature Compensation}

The temperature compensation should take into account that due to the converter switching mode, $L_{\mathrm{n}}$ and $L_{\text {ref }}$ are "alternatively" connected in series to the crystal in the oscillator in various sequences. The frequencies $f_{01}$ and $f_{02}$ given by Equations (8) and (10) have different times $t_{1}$ and $t_{2}$ (one after the other) depending on the digital control signals (wires $S_{1}-S_{3}$ ). This means that the subtraction in Equation (12) is not performed exactly point-to-point in time. It should be mentioned that the approach has some limitations in terms of the switching times and the time-speed of the events, i.e., temperature changes, whose effects can be cancelled. If these changes are sufficiently steep, temperature-related terms $\left(\Delta f_{0}\left(T_{1}\right)\right.$ and $\Delta f_{0}\left(T_{2}\right)$ in Equations (8) and (10) are not equal, so they are not fully counterbalanced in Equation (12)) may not be cancelled in Equation (8). As a result, the lineal first-order approximations of Equations (6) and (7) are no longer valid, which means that the influence of other terms is also non-negligible.

Frequency changes of the counter error $\Delta f_{\mathrm{c} 1}(t)$ and $\Delta f_{\mathrm{c} 2}(t)$ in Equations (6) and (7) are undefined, and presented here as a part of these equations. They, in fact, include both the counter measurement error and oscillator noise because it is very difficult to differentiate between the two. The frequency measurement errors can differ in every single measurement. Different oscillator noises (phase modulated (PM), jitter, and thermal Johnson) are all included in $\Delta f_{\mathrm{c}}(t)$ [21]. The switching mode method, first and foremost, compensates (considerably reduces) quartz crystal temperature influence. This influence is significantly greater than those of the noise and counter accuracy. 
The frequency measurement time depends on the gate time of the HM 8122 programmable counter and that of the LW software, as well as on the speed of the instrumentation IEEE 488 interface bus. To generate digital control signals $\left(S_{1}-S_{3}\right)$ and perform synchronous measurements, an additional electronic circuit NI myDAQ (Figure 3) was added. The switching signal switching between $f_{01}$ and $f_{02}$ is simultaneously used as an external signal triggering the HM 8122 programmable counter (frequency measurement (channel A and channel B). The counter synchronously measures the sequence frequency $f_{01}$ (channel $\mathrm{A}$ ) (the time of one measurement is determined by the counter gate time, which cannot be less than $1 \mathrm{~ms}$ ) and frequency $f_{\text {out }}$ (channel B). Similarly, the frequencies $f_{02}$ and $f_{\text {out }}$ are sequentially measured on the counter channel A and B in the next switching sequence, and LW software calculates the frequency difference between Equations (8) and (10). For every two frequencies measured by the counter, LW software calculates the frequency difference (Equation (12)).

The maximum variation temperature/time $(\Delta T / \Delta t)$ limit for which the compensation is still achieved is determined by the response times of the converter, LW software, and HM 8122 counter measurement time. Converter response $f_{0}$ versus inductance variation is determined by the eight-channel analog MUX switching time (the values for the ON and OFF mode are 59 ns and $60 \mathrm{~ns}$, respectively), and the low-pass filter time constant, which is $3 \mu$ s (passive LP filter first-order $\left.\left(\tau=R_{\mathrm{LP}} \cdot C_{\mathrm{LP}}\right)\right)$. If we take into account the response time of the two switches for one temperature-compensated inductance measurement, the converter response time is $\geqslant 10 \mu$ s. Due to LW software communication with the HM 8122 programmable counter and the time needed for the measurement (the time for one frequency measurement in the channel A or B is $1 \mathrm{~ms}$ ) of the two frequencies by the counter, the minimum response time is not less than $2.1 \mathrm{~ms}$. The maximum variation temperature/time $(\Delta T / \Delta t)$ limit for which the compensation is still achieved is determined by the dynamic frequency measurement error value during the time of both $f_{01}$ and $f_{02}$ signal period, i.e., within $2.1 \mathrm{~ms}$ (two consecutive measurements).

\subsection{Experimental Setup}

In this experiment, a prototype of a switching inductance-to-frequency converter circuit was constructed guaranteeing physically-stable conditions of the converter elements (Figure 4). A control switching logic was applied to the experimental oscillator circuit (eight-channel analog switch MUX) for the switching of the inductance $L_{1-7}, L_{\text {ref }}$ and capacitance (SPST analog switch) $C_{10}-C_{12}$ (Figure 3). The circuit also includes an OCXO oscillator (Figure 4), producing a frequency-stable reference rectangular signal, and pulse-width modulator (XOR logic gate) producing at the output a pulse-width modulated signal. A low-pass filter filters the frequency difference $f_{01}-f_{\mathrm{r}}$ in the first logic state of the switching $\left(S_{1}-S_{3}\right)$ and the frequency difference $f_{02}-f_{\mathrm{r}}$ in the second logic state of the switching $\left(\mathrm{S}_{1}-\mathrm{S}_{3}\right)$. The signal transformer transforms the voltage triangular signal to the voltage rectangular signal. The quartz oscillator is produced with SMD technology on $\mathrm{Al}_{2} \mathrm{O}_{3}$ because of good temperature stability and is placed into a metal housing. This design was used to achieve as stable parasitic capacitances and inductances in the circuits as possible. The quartz crystal $Q$ (Figure 3) used in the experiment was AT-cut crystal with a frequency change of $-2.5 \mathrm{ppm}$ at $\mathrm{T}=0^{\circ} \mathrm{C}$ and $1.5 \mathrm{ppm}$ at $T=50{ }^{\circ} \mathrm{C}$ depending on the reference temperature point $T_{\text {ref }}=25^{\circ} \mathrm{C}$. The data of the electrical quartz crystal $\left(f_{0}=4 \mathrm{MHz}\right)$ equivalent circuit elements are $R=10 \mathrm{Ohm}, C=25 \mathrm{fF}, L=64 \mathrm{mH}, C_{\mathrm{o}}=4 \mathrm{pF}$, and quality $Q=80 \mathrm{k}$. The values in the quartz crystal equivalent circuit were measured by an HP4194A impedance/gain-phase analyzer. Experimental circuits is connected to the NI myDAQ device with digital outputs for the control of the eight-channel analog switch MUX and a SPST analog switch. The NI myDAQ device is further connected to the computer via USB port. This enables automated changing of inductances $L_{1-7}$ at the input of the inductance-to-frequency converter and switching between $L_{1-7}$ and $L_{\text {ref }}$ and the setting of the converter sensitivity with capacitances $C_{10}-C_{12}$ (Figure 3). This mode of operation ensures stable parasitic capacitances and inductances, as well as repeatability of the experimental results $[28,29]$. 


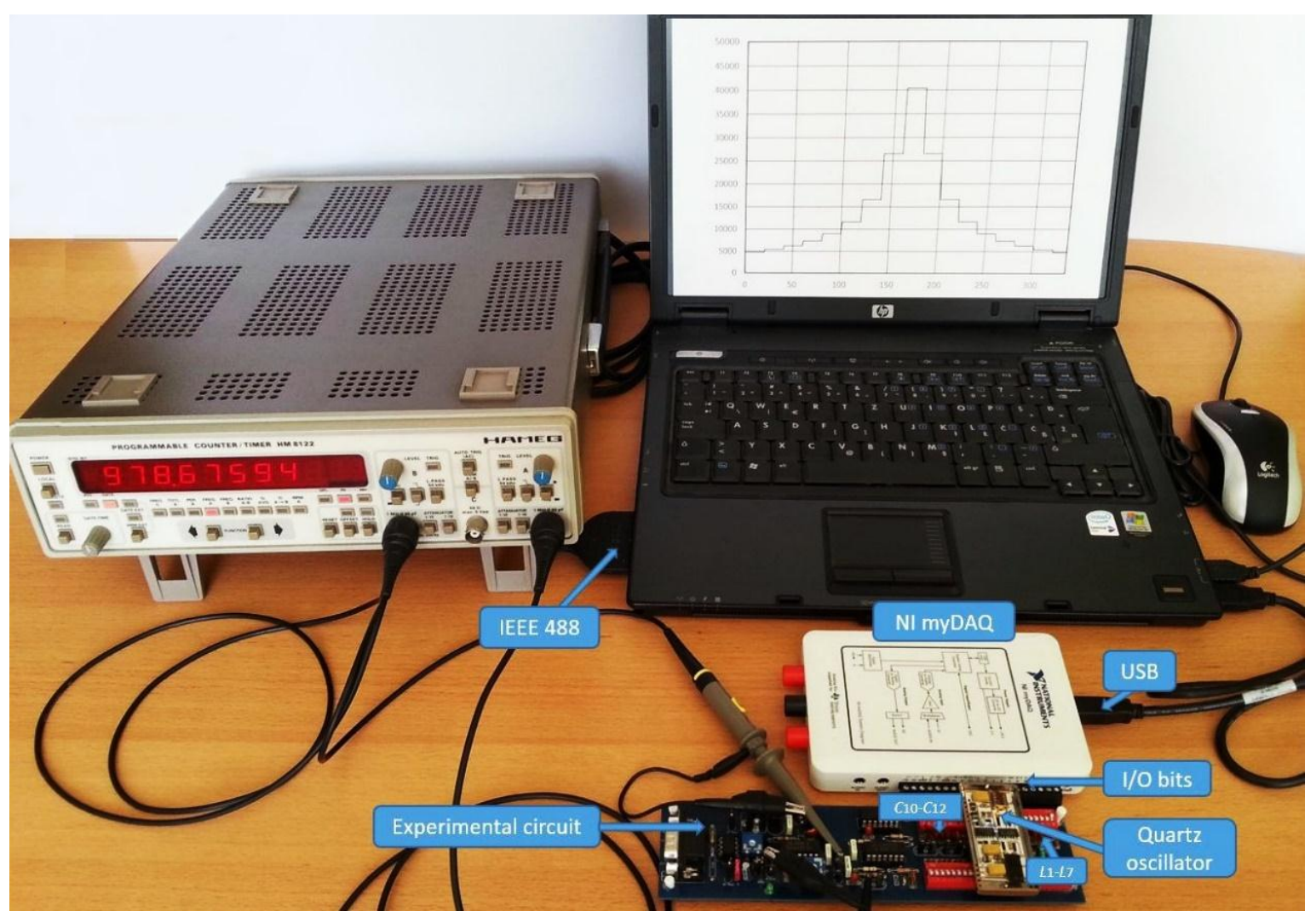

Figure 4. Experimental setup with experimental switching circuit.

The HM 8122 programmable counter is connected to the computer via the IEEE 488 interface bus. The measurement probes are connected directly to output pins $f_{\text {out }}$ and $f_{01-07}$ of the experimental circuit. Moreover, the LW software performs the switching of the oscillator frequency from $f_{01}$ to $f_{07}$ via NI myDAQ device, reads the frequency data for each $f_{0 \text { n }}$ from the counter via the IEEE 488 interface bus, and processes the data.

\section{Experimental Results}

To obtain good experimental results, a stable oscillator circuit in the inductance-to-frequency converter is of crucial importance. Therefore, the factors affecting frequency stability of the converter, such as wide operating temperature range, the use of various types of crystals, and drive level should be considered. Stability of the electronic circuit depends upon the quartz crystal temperature stability, the circuit type and quality of the elements. It is noteworthy to mention that an oscillator with a good start-up, i.e., with a reliable crystal oscillation during the start and later on, is a must.

\subsection{Inductance-Frequency Characteristics of the Converter}

Figure 5 shows oscillator's frequency characteristics $f_{01-07}$ with regard to the change of the inductance $L_{\mathrm{n}}=L_{1}$ to $L_{7}$ and a comparison of the characteristics for various $C_{10}-C_{12}$ (for the selection of various sensitivities) connected in parallel to the quartz crystal $Q$ (Figure 3). $L_{n}$ values are set by Ni myDAQ and are in steps of $0 \mu \mathrm{H}, 12 \mu \mathrm{H}, 25.4 \mu \mathrm{H}, 48 \mu \mathrm{H}, 75 \mu \mathrm{H}, 85 \mu \mathrm{H}, 95 \mu \mathrm{H}, 97.5 \mu \mathrm{H}$, and $100 \mu \mathrm{H}$. Within the switcher range $\left(L_{\mathrm{n}}=95-100 \mu \mathrm{H}\right)$ the inductance-frequency characteristics is almost linear. In the range $L_{\mathrm{n}}=95-100 \mu \mathrm{H}$, the capacitance $C_{10}=1 \mathrm{pF}$ records the sensitivity of $23.12 \mathrm{kHz} / \mu \mathrm{H}$, $C_{11}=3 \mathrm{pF}$ records the sensitivity of $31.58 \mathrm{kHz} / \mu \mathrm{H}$, and when $C_{12}=5 \mathrm{pF}$, the sensitivity is $39.26 \mathrm{kHz} / \mu \mathrm{H}$ (Figure 5). When only the quartz crystal's natural $C_{o}$ capacitance is present in the circuit, the sensitivity is $17.95 \mathrm{kHz} / \mu \mathrm{H}$. However, for this particular experiment, the capacitors $C_{10-12}$ and inductances $L_{\mathrm{n}}$ 
with tolerance of $0.1 \%$ were specially selected by the measurement with an HP 4194A impedance/gain phase analyzer.

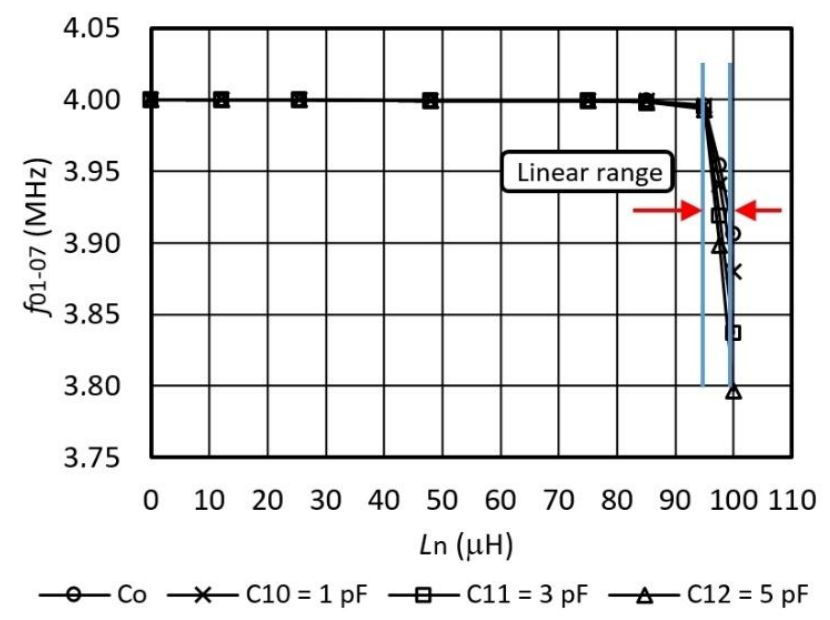

Figure 5. Inductance-frequency characteristics $f_{01-07}$ for inductance settings in steps of $0 \mu \mathrm{H}, 12 \mu \mathrm{H}$, $25.4 \mu \mathrm{H}, 48 \mu \mathrm{H}, 75 \mu \mathrm{H}, 85 \mu \mathrm{H}, 95 \mu \mathrm{H}, 97.5 \mu \mathrm{H}$, and $100 \mu \mathrm{H}$ (for different values of capacitance $C_{10}-C_{12}$ in parallel to the crystal at $\mathrm{T}=25^{\circ} \mathrm{C}$ ).

Figure 6 shows in greater detail inductance-frequency characteristics in the range of inductance-frequency switcher operation when the inductance is changed in the range $L_{\mathrm{n}}=95-100 \mu \mathrm{H}$. By using a switching multiplexer (and switching signals sent by wires $S_{1}-S_{3}$ ) (Figure 3), where various inductances $L_{\mathrm{n}}$ are switched, we get characteristics $f_{01-07}-f_{\mathrm{r}}$ and $f_{0 \text { Lref }}-f_{\mathrm{r}}$. Depicted are three different characteristics for three different capacitance values $C_{10}-C_{12}$, which are switched with the digital signals sent by wires $\mathrm{IN}_{1}-\mathrm{IN}_{3}$ (in the range 1,3 , and $5 \mathrm{pF}$ ) and characteristics $C_{0}$ (when the crystal does not have any additionally connected capacitance in parallel). Frequency difference $f_{0 \text { Lref }}-f_{\mathrm{r}}=5 \mathrm{kHz}$ is determined by the reference inductance $L_{\text {ref }}$ (Figure 3 ) and the OCXO reference oscillator, and serves for the transformation of the signal in the lower frequency range and for the temperature compensation. Frequency signal $f_{\text {out }}$ represents the time duration of one switching $f_{01-07}-f_{\mathrm{r}}$ and in the next one $f_{0 \text { Lref }}-f_{\mathrm{r}}$. The difference of frequencies $\left(f_{01-07}-f_{\mathrm{r}}\right)-\left(f_{0 \text { Lref }}-f_{\mathrm{r}}\right)$ in the sequence of two switchings is then calculated by the computer and represents the final switcher's inductance-frequency characteristics. Thus, the most sensitive characteristics are obtained at $C_{12}=5 \mathrm{pF}$. The greatest frequency difference of $\left(f_{01-07}-f_{\mathrm{r}}\right)-\left(f_{0 \text { Lref }}-f_{\mathrm{r}}\right)$ at the sensitivity of $39.26 \mathrm{kHz} / \mu \mathrm{H}$ is $\cong 200 \mathrm{kHz}$.

Figure 7 shows the measurement of the hysteresis error if the inductance $L_{n}$ is set in steps between $0 \mu \mathrm{H}$ and $100 \mu \mathrm{H}$ (frequencies $f_{01-07 u p}$ ) and back from $100 \mu \mathrm{H}$ to $0 \mu \mathrm{H}$ (frequencies $f_{07-01 \text { down }}$ ). The influences of the temperature, oscillator element aging, and the influences of parasitic capacitances are reduced to a minimum because in the frequency difference $\left(\left(f_{01-07 u p}-f_{\mathrm{r}}\right)-\left(f_{0 \text { Lref }}-f_{\mathrm{r}}\right)\right)-\left(\left(f_{07-01 \text { down }}\right.\right.$ $\left.\left.-f_{\mathrm{r}}\right)-\left(f_{0 \text { Lref }}-f_{\mathrm{r}}\right)\right)$ remains only $\left(f_{01-07 \mathrm{up}}-f_{07-01 \text { down }}\right)$, which represents the hysteresis measurement for three capacitances $\left(C_{10}, C_{11}\right.$ and $\left.C_{12}\right)$. Also shown are the hysteresis characteristics for $C_{0}$ only, without any additionally-connected capacitances in parallel to the quartz crystal. The hysteresis influence on Figure 7 is visible because, by increasing the value of inductance $L_{n}$, the hysteresis (frequency difference $\left(f_{01-07 u p}-f_{07-01 \text { down }}\right)$ ) difference increases. There are three regions $\mathrm{A}, \mathrm{B}$, and $\mathrm{C}$ shown in Figure 7 . In the (A) region, the hysteresis frequency difference for all three capacitance values is $C_{10-12} \leqslant 1 \cdot 10^{-12}$, in the (B) region of inductance $L_{n}=25-75 \mu \mathrm{H}$, the frequency difference is $\cong 1 \times 10^{-12}$ and in the $(\mathrm{C})$ region $\left(L_{\mathrm{n}}=75-99 \mu \mathrm{H}\right)$ there is a maximum value (Max) of frequency difference at $L_{\mathrm{n}}=99 \mu \mathrm{H}$ and it is $9 \times 10^{-12}$ at the value of capacitance $C_{12}=5 \mathrm{pF}$ (at maximum sensitivity of the inductance-to-frequency switcher). 


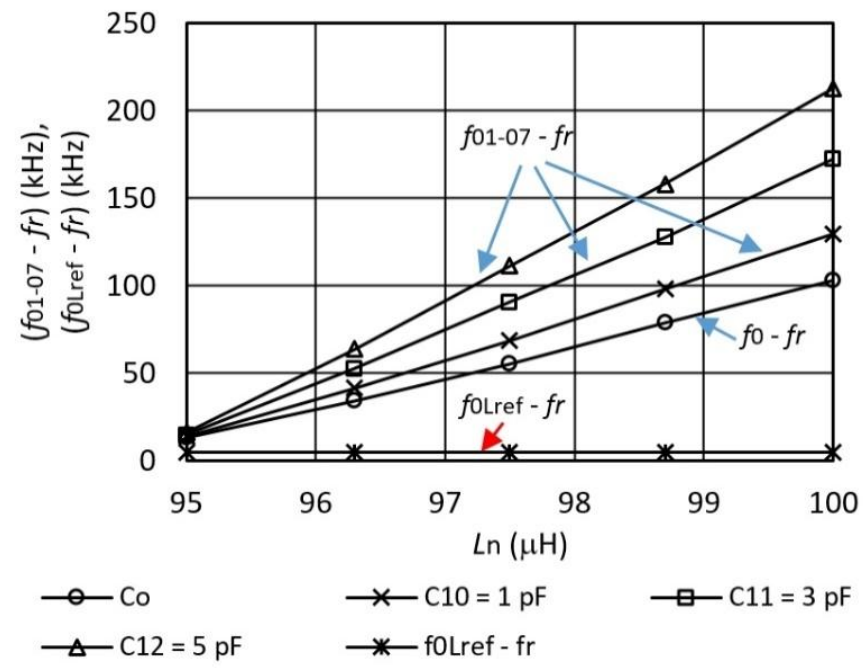

Figure 6. Characteristics $f 0-f_{\mathrm{r}}, f 01-07-f_{\mathrm{r}}$ (depending on $L_{\mathrm{n}}$ ) and $f_{0 \text { Lref }}-f_{\mathrm{r}}=5 \mathrm{kHz}$ acquired with the alternate multiplexer switching at different capacitances $C_{10-12}=1,3$, and $5 \mathrm{pF}$ at $T=25^{\circ} \mathrm{C}$ ).

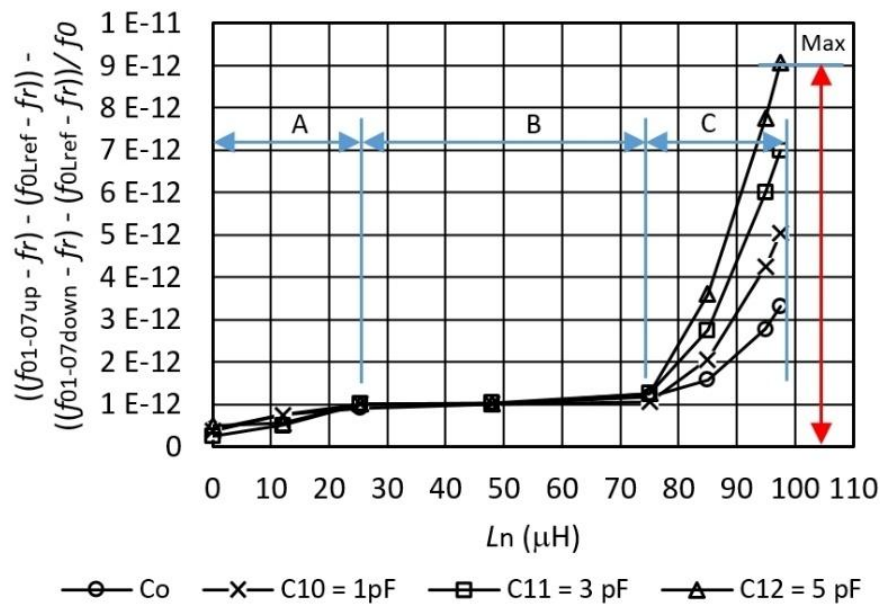

Figure 7. Hysteresis measurement error of the inductance-to-frequency converter depending on the $L_{\mathrm{n}}=0-99 \mu \mathrm{H}$ and capacitor value from $C_{10-12}=1-5 \mathrm{pF}$, and switching frequency $f_{\text {switch }}=1 \mathrm{~Hz}$ between $L_{\mathrm{n}}$ and $L_{\text {ref. }}$

\subsection{Temperature/Frequency Stability of the Switching Converter}

If the converter is influenced by a temperature changing from 0 to $50^{\circ} \mathrm{C}$, the switching mode extended dynamic frequencies change $f_{01-07}-f_{\mathrm{r}}$ and $f_{0 \text { Lref }}-f_{\mathrm{r}}$ is approximately the same, and it decreases as shown in Figure 8 . For every state $f_{01-07}-f_{\mathrm{r}}$ and $f_{0 \text { Lref }}-f_{\mathrm{r}}$ consecutive measurements were made. The temperature control was performed by Weiss SB1 160 programmable climate chamber (Weiss Umwelttechnik GmbH, Stuttgart, Germany). The dotted trend line points to the change of direction of both frequency differences (decreases). These results demonstrate that the temperature influence on the frequency differences $f_{01-07}-f_{\mathrm{r}}$ and frequency $f_{0 \mathrm{Lref}}-f_{\mathrm{r}}$ (in the time span between 0 and $240 \mathrm{~s}$ ) changes the frequency difference in the same size class (Diff 1, Diff 2). Given that the frequency change is almost the same at $0{ }^{\circ} \mathrm{C}$ and $50{ }^{\circ} \mathrm{C}$ (Figure 8), the temperature influence is reduced to minimum. The temperature in the immediate vicinity of the crystal was measured by the NI USB-TC01 thermocouple measurement device. The frequency shift shown in Figure 8 between $f_{01-7}-f_{\mathrm{r}}$ and $f_{0 \text { Lref }}-f_{\mathrm{r}}$ depends on the difference between $L_{\mathrm{n}}$ and $L_{\mathrm{ref}}\left(L_{\mathrm{n}}=95.01 \mu \mathrm{H}\right.$ and $\left.L_{\mathrm{ref}}=95 \mu \mathrm{H}\right)$. 


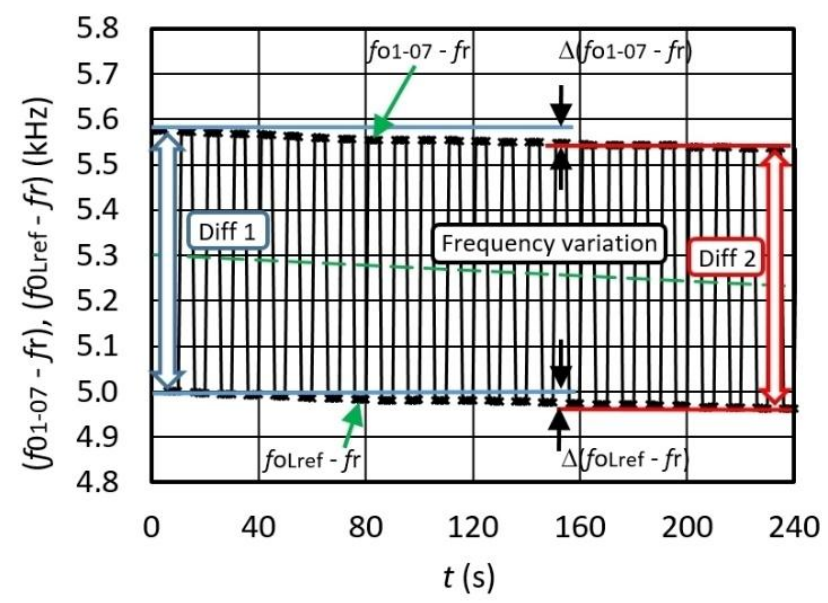

Figure 8. Extended temperature dynamic (switching mode) frequency instability for $f_{01-07}-f_{\mathrm{r}}$ and $f_{0 \text { Lref }}-f_{\mathrm{r}}$ (sensitivity is $31.58 \mathrm{kHz}$ ) (temperature change from 0 to $50{ }^{\circ} \mathrm{C}$ ).

Figure 9 illustrates the frequency variation for $\Delta f_{\text {out }}=\Delta\left(\left(f_{01-07}-f_{\mathrm{r}}\right)-\left(f_{\text {oLref }}-f_{\mathrm{r}}\right)\right) / f_{0}$ at the frequency difference $\cong 558 \mathrm{~Hz}\left(f_{0 \mathrm{Lref}}-f_{\mathrm{r}}=5 \mathrm{kHz}\right.$ (Figure 8)) determined by the fixed values $L_{\mathrm{n}}$ and $L_{\mathrm{ref}}\left(L_{\mathrm{n}}=95.01 \mu \mathrm{H}, L_{\mathrm{ref}}=95 \mu \mathrm{H}\right)$ in the time span $t=0-240 \mathrm{~s}$ during the temperature change $0-50{ }^{\circ} \mathrm{C}$. Deduction of frequencies (between $f_{01-7}-f_{\mathrm{r}}$ and $f_{0 \text { Lref }}-f_{\mathrm{r}}$ ) in relation to the synchronization signal is performed by LW software in sequence at different times $t_{\mathrm{e}}$. The differences between $f_{01-7}-f_{\mathrm{r}}$ and $f_{0 \text { Lref }}-f_{\mathrm{r}}$ show a high-frequency dynamic stability in the range $\pm 7 \times 10^{-13}$ (as pointed by arrows on Figure 9).

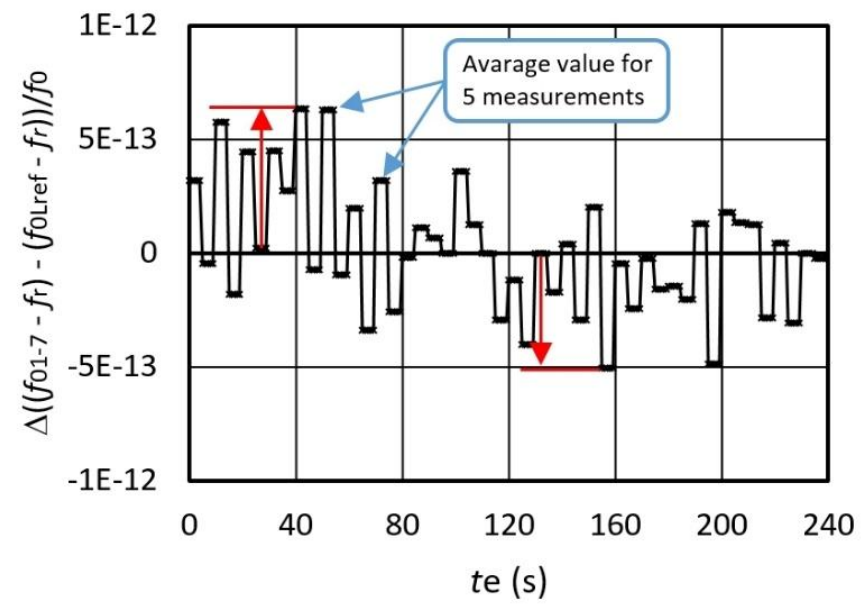

Figure 9. Output frequency variation of $\Delta f_{\text {out }}=\Delta\left(\left(f_{01-07}-f_{\mathrm{r}}\right)-\left(f_{0 \text { Lref }}-f_{\mathrm{r}}\right)\right) / f_{0}$ during the change of temperature from 0 to $50^{\circ} \mathrm{C}\left(L_{\mathrm{n}}=95.01 \mu \mathrm{H}, L_{\text {ref }}=95 \mu \mathrm{H}\right)$.

\section{Discussion}

This switching method makes possible a high-precision measurement of accurate characteristics and hysteresis of the inductance-to-frequency converter through experimental automated approach.

Due to almost instant comparison (that lasts only a few milliseconds) of the measured inductance with the reference one, the environment temperature remains virtually unchanged. In case of two consecutive inductance measurements and the conversion to frequency (at dynamic environment temperature changes), the temperature influence is also reduced to a minimum, as shown by the results. The frequency difference of two consecutive switchings namely lasts only a few milliseconds (Figures 8 and 9). An automated approach of changing inductance with the help of an analog MUX 
switch (Figure 3) ensures that during the switchings there are no additional changing influences of parasitic inductances and capacitances. The SPST analog switch plays a similar role, changing the load capacitance and, as a result, the sensitivity of the inductance-to-frequency converter.

If the output converter frequency sensitivity of $39.26 \mathrm{kHz}$ is in the range $L_{\mathrm{n}}=95-100 \mu \mathrm{H}$ at $C_{12}=5 \mathrm{pF}$ (Figure 6), the supply voltage stability is $5 \mathrm{~V} \pm 0.01 \mathrm{~V}$, the HM8122 counter accuracy of $\pm 5 \times 10^{-9}$ (through the working temperature range from 10 to $40^{\circ} \mathrm{C}$, and the frequency reference $f_{\mathrm{r}}$ stability is $0.005 \mathrm{ppm}$, then the frequency stability at the output is $f_{\text {out }}= \pm 7 \times 10^{-13}$, which gives the converter a resolution of $\pm 2 \mathrm{pH}$ in the temperature range between 0 and $50{ }^{\circ} \mathrm{C}$. Thus, the output frequency stability proves the applicability of the automated hysteresis measurement method of the inductance-to-frequency converter in the $\mathrm{pH}$ range.

\section{Conclusions}

The proposed measurement method enables temperature compensation and stable hysteresis measurement of an inductance-to-frequency converter. The greatest advantage of the proposed method is that the programmable timing control device allows for the selection of different oscillating frequencies. The method simultaneously compensates not only for the stray capacitances and inductances, but also for the crystal's natural temperature characteristics. Moreover, it enables additional software-managed decreasing of the inductance and any parasitic capacitance minimizing external influences.

The hysteresis measurement results clearly show that the oscillator switching method for high-precision inductance-to-frequency conversion opens up new possibilities through the compensation of the main oscillating element self-temperature and minimization of other frequency variation influences (such as aging, the influence of the supply voltage on the oscillating circuit, as well as the reference frequency temperature instability). The method can be applied in many different fields, such as nanorobotics, mechatronics, magnetic material properties measurement, biosensor technology, as well as in specific fields of physics.

Acknowledgments: The authors are grateful to Slovenian National Science Foundation for partial support of this work though projects under Grant P2-0028.

Author Contributions: The presented work was carried out in collaboration between both authors. Vojko Matko developed the switching measurement method for hysteresis measurement, software and wrote the manuscript while Miro Milanović analyzed the temperature compensation and supervised the work.

Conflicts of Interest: The authors declare no conflict of interest.

\section{References}

1. Kenton, B.J.; Leang, K.K. Design and control of a three-axis serial-kinematic high-bandwidth nanopositioner. IEEE/ASME Trans. Mechatron. 2012, 17, 356-369. [CrossRef]

2. Noth, K.T.; Ryu, U.C.; Lee, Y.W. Compact and wide range polarimetric strain sensor based on polarization-maintaining photonic crystal fiber. Sens. Actuators A Phys. 2014, 213, 89-93.

3. Chen, S.C.; Le, D.K.; Nguyen, V.S. Inductive displacement sensors with a notch filter for an active magnetic bearing system. Sensors 2014, 14, 12640-12657. [CrossRef] [PubMed]

4. Guo, Y.X.; Shao, Z.B.; Li, T. An analog-digital mixed measurement method of inductive proximity sensor. Sensors 2016, 16, 30. [CrossRef] [PubMed]

5. Xiong, J.; Li, C.; Jia, P.; Chen, X.; Zhang, W.; Liu, J.; Xue, C.; Tan, Q. An Insertable passive lc pressure sensor based on an alumina ceramic for in situ pressure sensing in high-temperature environments. Sensors 2015, 15, 21844-21856. [CrossRef] [PubMed]

6. Li, C.; Tan, Q.; Jia, P.; Zhang, W.; Liu, J.; Xue, C.; Xiong, J. Review of research status and development trends of wireless passive LC resonators for harsh environments. Sensors 2015, 15, 13097-13109. [CrossRef] [PubMed]

7. Chiou, J.A.; Chen, S. Thermal hysteresis and voltage shift analysis for differential pressure sensors. Sens. Actuators A Phys. 2007, 135, 107-112. [CrossRef] 
8. García, A.; Morón, C.; Tremp, E. Magnetic sensor for building structural vibration. Sensors 2014, 14, $2468-2475$. [CrossRef] [PubMed]

9. Jeranče, N.; Bednar, N.; Stojanović, G. An Ink-Jet Printed Eddy Current Position Sensor. Sensors 2013, 13, 5205-5219. [CrossRef] [PubMed]

10. García, M.H.; Barrera, D.; Amat, R.; Kurlyandskaya, G.V.; Sales, S. Magnetic actuator based on giant magnetostrictive material Terfenol-D with strain and temperature monitoring using FBG optical sensor. Measurement 2016, 80, 201-206. [CrossRef]

11. Matko, V.; Jezernik, K. New quartz oscillator switching method for nano-henry range inductance measurements. Sensors 2012, 12, 3105-3117. [CrossRef] [PubMed]

12. Arnau, A. A review of interface electronic systems for AT-cut quartz crystal microbalance applications in liquids. Sensors 2008, 8, 370-411. [CrossRef]

13. Ferrari, M.; Ferrari, V.; Marioli, D.; Taroni, A.; Suman, M.; Dalcanale, E. In-liquid sensing of chemical compounds by QCM sensors coupled with high-accuracy ACC oscillator. IEEE Trans. Instrum. Meas. 2006, 55, 828-834. [CrossRef]

14. Gagnepain, J.J. Sensitivity of quartz oscillator to the environment: Characterization methods and pitfalls. IEEE Trans. Ultrason. Ferroelect. Freq. Cont. 1990, 37, 347-354. [CrossRef] [PubMed]

15. Schrüfer, E. Quartz as a frequency reference. In Electrical Measurement; Carl Hanser Verlag: Munich, Germany, 1992; pp. 405-412.

16. Brice, J.C. Crystals for quartz resonators. Rev. Mod. Phys. 1985, 57, 105-138. [CrossRef]

17. Meeker, T.R. Theory and Properties of Piezoelectric Resonators and Waves. In Precision Frequency Control; Academic Press: Waltham, MA, USA, 2007; Volume 1, pp. 47-119.

18. Yeh, C.A.; Lai, Y.S. Digital pulsewidth modulation technique for a synchronous buck converter to reduce switching frequency. IEEE Trans. Ind. Electron. 2012, 59, 550-561. [CrossRef]

19. Zhao, Z.; Lai, J.S.; Cho, Y. Dual-mode double-carrier-based sinusoidal pulse width modulation inverter with adaptive smooth transition control between modes. IEEE Trans. Ind. Electron. 2013, 60, 2094-2103. [CrossRef]

20. Kiatsookkanatorn, P.; Sangwongwanich, S. A unified PWM method for matrix converters and its carrier-based realization using dipolar modulation technique. IEEE Trans. Ind. Electron. 2012, 59, 80-92. [CrossRef]

21. Driscoll, M.M. Oscillator AM-to-FM Noise conversion due to the dynamic frequency-drive sensitivity of the crystal resonator. IEEE FCS 2008, 672-676. [CrossRef]

22. Filler, R.L.; Vig, J.R. Long-term aging of the oscillators. IEEE Trans. Ultrason. Ferroelectr. Freq. Cont. 1992, 39, 241-249.

23. Matko, V.; Jezernik, K. Greatly improved small inductance measurement using quartz crystal parasitic capacitance compensation. Sensor 2010, 10, 3954-3960. [CrossRef] [PubMed]

24. Wu, I.C.; Lo, C.W.; Fong, K.L. Method and Apparatus for a Crystal Oscillator to Achieve Fast Start-Up Time, Low Power and Frequency Calibration. US Patent 7348861 B1, 25 March 2008.

25. Ruslan, R.I.; Satoh, T.; Akitsu, T. Short-term Stability in the Intermediate Region between Quartz Crystal Oscillation and LC Oscillation. IEEJ Trans. Electr. Electron. Eng. 2012, 7, 46-51. [CrossRef]

26. Rutman, J. Characterization of phase and frequency instabilities in precision frequency sources. Proc. IEEE 1978, 66, 1048-1075. [CrossRef]

27. Langfelder, G.; Caspani, A.; Tocchio, A. Design criteria of low-power oscillators for consumer-grade MEMS resonant sensors. IEEE Trans. Ind. Electron. 2014, 61, 555-566. [CrossRef]

28. Wang, S.; Lee, F. Analysis and applications of parasitic capacitance cancellation techniques for EMI suppression. IEEE Trans. Ind. Electron. 2010, 57, 3109-3117. [CrossRef]

29. Kao, P.; Allara, D.; Tadigadapa, S. Fabrication and performance characteristics of high-frequency micromachined bulk acoustic wave quartz resonator arrays. Meas. Sci. Technol. 2009. [CrossRef]

(C) 2016 by the authors; licensee MDPI, Basel, Switzerland. This article is an open access article distributed under the terms and conditions of the Creative Commons Attribution (CC-BY) license (http://creativecommons.org/licenses/by/4.0/). 